\title{
Virtual anatomy and pathoanatomy: Cinematic Rendering of a proximal humerus fracture
}

\author{
Alexander Firmötz ${ }^{1}$ and Franz A Fellner ${ }^{1,2 *}$ \\ ${ }^{1}$ Central Radiology Institute, Kepler University Hospital, Medical Faculty of the Johannes Kepler University, Linz, Austria \\ ${ }^{2}$ Medical Faculty of the Friedrich-Alexander-University of Erlangen-Nürnberg, Erlangen, Germany
}

\section{Case report}

We report a 71-year old patient, who suffered from intense shoulder pain after he fell. The initial plain radiograph revealed a complex fracture of the left proximal humerus (Figure 1).

As part of the preoperative planning a CT scan of the left shoulder was acquired. In addition to the standard reconstructions, the affected area was visualized using Cinematic Rendering to gain a threedimensional display (Figure 2).

The operative procedure was performed without any complications and the multiple bone fragments could be reponed successfully. Figure 3 shows the follow-up plain radiograph after osteosynthesis. A few days after the operation the patient was discharged in good general condition.

\section{Discussion}

Digital post-processing techniques for CT and MR examinations, such as multiplanar reconstructions (MPR), maximum intensity projections (MIP), or Volume Rendering (VR) have been established for decades. In this context Cinematic Rendering is a novel rendering technology [1].

In comparison to Volume Rendering, Cinematic Rendering can create more photorealistic displays of anatomical and pathoanatomical structures [1,2], when using CT, especially for osseous structures, since these show a high contrast in X-ray and CT [3].

This lifelike imaging is based on complex light models including different physical effects of light rays. The result is a complex simulation of light and shadow improving the perception and furthermore the understanding of those structures [4,5].

Due to the clear presentation of the situs, the time required for interpretation during the preoperative assessment possibly may be reduced and the quality of the preoperative planning improved [6].

Besides clinical use, Cinematic Rendering is now also used in medical education. Anatomy courses based on Cinematic Rendering can be designed in a more versatile way compared to conventional dissection courses. The option of showing anatomical variants or pathological changes is an excellent addition to conventional anatomy lessons [6-9].

\section{Disclosure}

No conflicts of interest. No grants or financial supports.
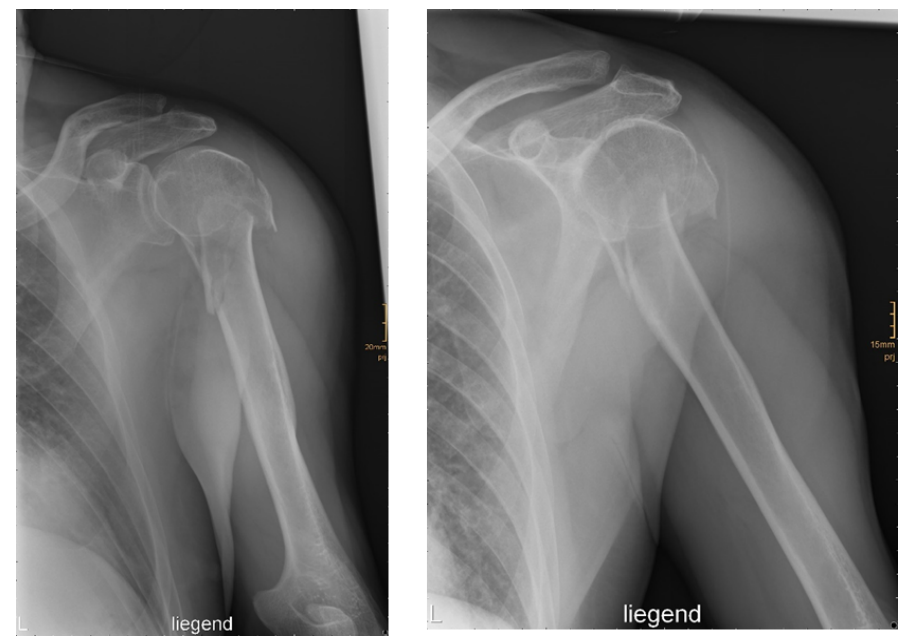

Figure 1. Plain radiograms of the proximal humerus fracture
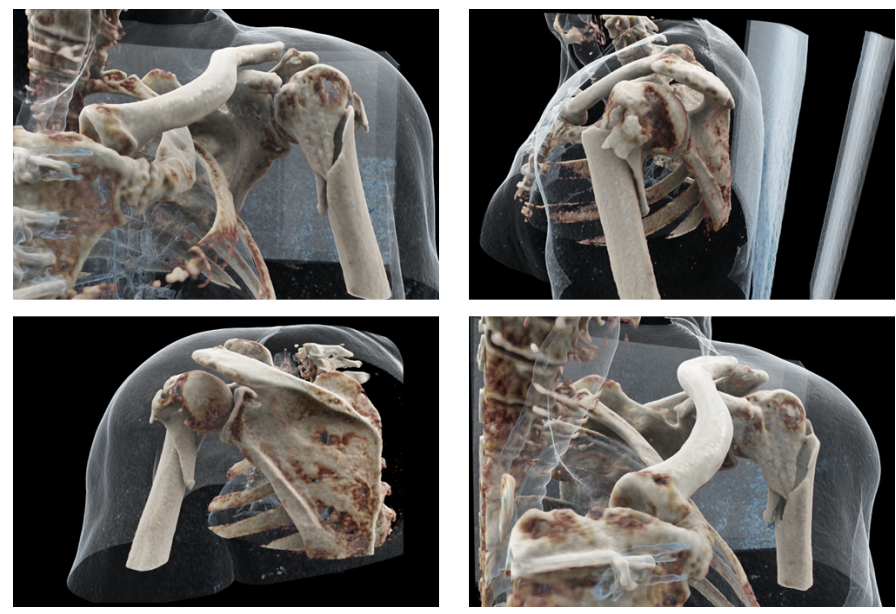

Figure 2. Three-dimensional visualization of the preoperative CT scan using Cinematic Rendering

${ }^{\star}$ Correspondence to: Alexander Firmötz, Central Radiology Institute, Kepler University Hospital, Medical Faculty of the Johannes Kepler University, Linz, Austria, E-mail: alexander.firmoetz@kepleruniklinikum.at

Key words: virtual anatomy, virtual pathoanatomy, cinematic rendering, proximal humerus fracture

Received: February 15, 2020; Accepted: March 10, 2020; Published: March 12, 2020 

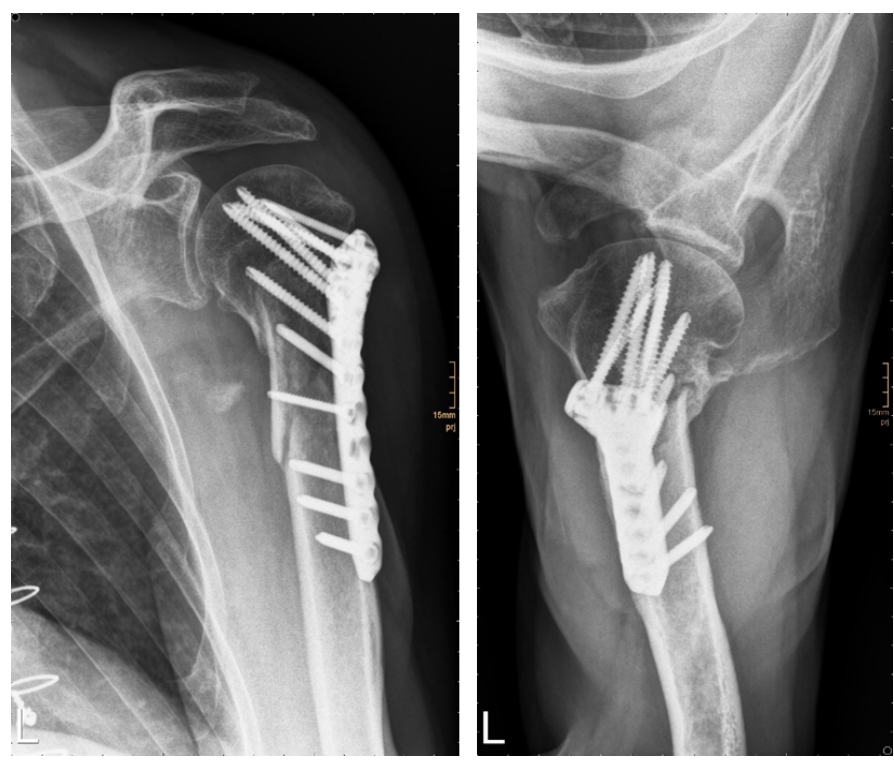

Figure 3. Follow-up plain radiograph after osteosynthesis of the proximal humerus

\section{References}

Fellner FA(2016) Introducing cinematic rendering: A novel technique for postprocessing medical imaging data. J Biomedical Science and Engineering 9: 170-175.

2. Comaniciu D, Engel K, Georgescu B, Mansi T (2016) Shaping the future through innovations: from medical imaging to precision medicine. Med Image Anal 33: 19-26.

3. Dappa E, Higashigaito K, Fornaro J, Leschka S (2016) Cinematic rendering - an alternative to volume rendering for $3 \mathrm{D}$ computed tomography imaging. Insights Imaging 7: 849-856.

4. Eid M, De Cecco CN, Nance JW, Caruso D, Albrecht, MH, et al. (2017) Cinematic Rendering in CT: A Novel, Lifelike 3D Visualization Technique. American Journal of Roentgenology 209(2): 370-379. [Crossref]

5. Langer MS, Bülthoff HH (2000) Depth discrimination from shading under diffuse lighting. Perception 29(6): 649-660. [Crossref]

6. Elshafei M, Binder J, Baecker J, Brunner M (2019) Comparison of Cinematic Rendering and Computed Tomography for Speed and Comprehension of Surgical Anatomy. JAMA Surg 154(8): 738-744. [Crossref]

7. Fellner FA, Berger F, Fellner C, Kremer C, Hortner H, et al. (2016) Volume rendering of medical imaging data for the general public. J Health Med Informat 7: 213.

8. Fellner FA, Engel K, Kremer C (2017) Virtual Anatomy: The dissecting theatre of the future - implementation of cinematic rendering in a large $8 \mathrm{~K}$ high-resolution projection environment. J Biomedical Science and Engineering 10: 367-375.

9. Binder J, Krautz C, Engel K, Grützmann R, Fellner FA, et al. (2019) Leveraging medical imaging for medical education - a cinematic rendering-featured lecture. An Anat 222: 159-165. [Crossref]

Copyright: (C2020 Vanderweckene P. This is an open-access article distributed under the terms of the Creative Commons Attribution License, which permits unrestricted use, distribution, and reproduction in any medium, provided the original author and source are credited. 